University of Nebraska - Lincoln

DigitalCommons@University of Nebraska - Lincoln

Papers in Veterinary and Biomedical Science

Veterinary and Biomedical Sciences,

Department of

January 2008

\title{
The interaction between KSHV RTA and cellular RBP-JK and their subsequent DNA binding are not sufficient for activation of RBP- JK
}

Anil Papugani

University of Nebraska - Lincoln

Tricia Coleman

University of Nebraska - Lincoln

Clinton J. Jones

University of Nebraska - Lincoln, cjones2@unl.edu

Luwen Zhang

University of Nebraska - Lincoln, Izhang2@unl.edu

Follow this and additional works at: https://digitalcommons.unl.edu/vetscipapers

Part of the Veterinary Medicine Commons

Papugani, Anil; Coleman, Tricia; Jones, Clinton J.; and Zhang, Luwen, "The interaction between KSHV RTA and cellular RBP-JK and their subsequent DNA binding are not sufficient for activation of RBP-JK" (2008). Papers in Veterinary and Biomedical Science. 85.

https://digitalcommons.unl.edu/vetscipapers/85

This Article is brought to you for free and open access by the Veterinary and Biomedical Sciences, Department of at DigitalCommons@University of Nebraska - Lincoln. It has been accepted for inclusion in Papers in Veterinary and Biomedical Science by an authorized administrator of DigitalCommons@University of Nebraska - Lincoln. 


\title{
The interaction between KSHV RTA and cellular RBP-Jא and their subsequent DNA binding are not sufficient for activation of RBP-JK
}

\author{
Anil Papugani ${ }^{1}$, Tricia Coleman ${ }^{2, \dagger}$, Clinton Jones ${ }^{2,3}$, and Luwen Zhang ${ }^{1,2, *}$ \\ ${ }^{1}$ School of Biological Sciences, University of Nebraska-Lincoln, Lincoln, NE 68588, USA \\ ${ }^{2}$ Nebraska Center for Virology, University of Nebraska-Lincoln, Lincoln, NE 68588, USA \\ ${ }^{3}$ Department of Veterinary Biomedical Sciences, University of Nebraska-Lincoln, Lincoln, NE 68588, USA \\ ${ }^{*}$ Corresponding author: E141 Beadle Center, Nebraska Center for Virology, University of Nebraska-Lincoln, \\ 1901 Vine St., Lincoln, NE 68588, USA; tel. 402472 5905; fax 4024728722. \\ $\dagger$ Present address: Abbott Laboratories, 200 Abbott Park Road, Abbott Park, IL 66064, USA.
}

\begin{abstract}
Kaposi's sarcoma-associated herpesvirus (KSHV) replication and transcription activator (RTA) is necessary and sufficient for the switch from KSHV latency to lytic replication. RTA activates promoters by several mechanisms. RTA can bind to sequences in viral promoters and activate transcription. In addition, RTA interacts with the cellular recombination signal sequence-binding protein-J kappa (RBP-JK), a transcriptional re-

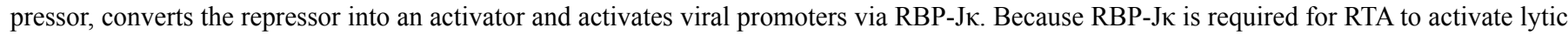
replication, it is important to understand how RTA cooperates with RBP-Jк protein to activate KSHV lytic replication program. Previously, we identified an RTA mutant, RTA-K152E, which has a defect in its direct DNA-binding activity. In this report, the effect of the mutant RTA on

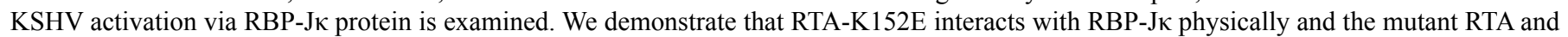
RBP-JK complex binds to target DNA properly in vivo and in vitro. However, the complex of RTA-K152E and RBP-JK does not activate transcription. Furthermore, the RTA mutant (RTA-K12E) inhibits cellular Notch-mediated RBP-JK activation. These data collectively suggest that the complex between KSHV RTA and cellular RBP-J $\kappa$ and the subsequent DNA binding by the complex are not sufficient for the activation of RBP-J $\kappa$ protein. Other factor(s) whether additional cofactor(s) in the complex or the intrinsic conformation of RTA, are predicted to be required for the activation of RBP-Jא protein by RTA.
\end{abstract}

Keywords: KSHV, RTA, RBP-Jк, Lytic replication, HHV8, PEL

\section{Introduction}

Kaposi's sarcoma (KS)-associated herpesvirus (KSHV), also known as human herpesvirus 8 (HHV-8), is a gamma herpesvirus. It is believed to be the etiological agent of KS (Chang et al., 1994; Chang and Moore, 1996; Moore and Chang, 2001). $\mathrm{KSHV}$ is also implicated in the pathogenesis of AIDS-associated primary effusion lymphoma (PEL, also called body cavity-based lymphoma (BCBL)) and a lymphoproliferative disorder known as multicentric Castleman's disease (Dourmishev et al., 2003; Moore and Chang, 2001; West and Wood, 2003).

As other herpesviruses, KSHV goes through both latency and lytic replication cycles. The expression of the KSHV replication and transcription activator (RTA) is necessary and sufficient for the switch from latency to lytic replication
(Dourmishev et al., 2003; West and Wood, 2003). RTA is an immediate early gene (Sarid et al., 1998; Sun et al., 1999; Zhu et al., 1999) and a sequence-specific DNA-binding protein. A number of RTA-responsive elements (RRE) were identified in the transcriptional regulatory regions of different subsequently expressed viral genes (Gradoville et al., 2000; Lukac et al., 1999; Sakakibara et al., 2001; Saveliev et al., 2002; Sun et al., 1998; Sun et al., 1999; Zhu et al., 1999).

In addition to direct DNA binding, the ability of RTA to interact with other factors appears to be necessary for activating its transcriptional potential (Gwack et al., 2001; Gwack et al., 2002; Liao et al., 2003; Wang et al., 2001). One of the interacting factors is the cellular recombination signal sequencebinding protein-J kappa (RBP-Jк) (also known as CBF-1 and CSL) (Carroll et al., 2006; Liang et al., 2002; Liang and 
Ganem, 2003). RBP-JK is a sequence-specific DNA-binding protein and a transcriptional repressor that is involved in the Notch signaling pathway.

The Notch signaling pathway regulates cell fate in a variety of organisms (Bray, 2006; He and Pear, 2003). Interaction of Notch receptors (Notch) with their ligands leads to cleavage of the Notch intracellular domain (NICD), which leads to nuclear localization of NICD. In the nucleus, NICD associates with RBP-JK and converts RBP-JK from a transcriptional repressor to an activator. Furthermore, the NICD-RBP-Jא complex activates expression of specific target genes (Bray, 2006; He and Pear, 2003).

KSHV RTA can directly bind to RBP-Jא and the RTARBP-JK complex binds to DNA and activates transcription of KSHV promoters (Carroll et al., 2006; Liang et al., 2002; Liang and Ganem, 2003). The KSHV genome contains numer-

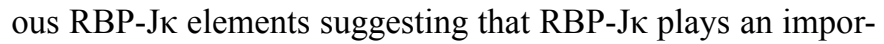
tant role in viral transcription. The interaction between RTA and RBP-JK is essential for the switch from viral latency to lytic replication in rodent cells (Liang and Ganem, 2003). However, it is not clear if interaction between RTA and RBP$\mathrm{J}_{\kappa}$ is the only event that is necessary for activating KSHV lytic gene expression.

Previously, we have identified a novel function of RTA, i.e., RTA binds to interferon-stimulated response elements (ISRE) and activate certain cellular interferon-stimulated genes (ISG) (Zhang et al., 2005). In addition, a region in RTA DNA-binding domain has been identified with certain sequence similarity to the DNA-binding domains of interferon regulatory factor (IRF) family. Mutation in one conserved amino acid within this region (RTA-K152E) reduces the ability of RTA to bind to ISRE as well as other RREs. Furthermore, the RTA-K152E fails to activate RTA-responsive promoters and to induce viral lytic gene expression (Zhang et al., 2005).

In this report, we have further characterized the RTAK152E mutant in terms of activating the cellular RBP-Jא protein. The mutant RTA is able to interact with RBP-Jк physically and the RTA-K152E-RBP-JK complex is able to bind to DNA in vitro and in vivo. However, the mutant failed to activate RBP-J $\kappa$ protein. Thus, the complex between KSHV RTA and cellular RBP-JK and their subsequent DNA binding are not sufficient for the activation of RBP-JK protein.

\section{Materials and methods}

\subsection{Plasmids and antibodies}

Expression plasmids of FLAG-tagged RTA and mutant RTAK152E were previously described (Zhang et al., 2005). The HA-tagged RBP-JK expression plasmid was a gift from Dr. Paul Ling (Baylor College of Medicine). FLAG-tagged RBP$\mathrm{J} \kappa$ expression plasmid and the reporter construct containing $3 \times$ RBP-J $\mathrm{K}$ binding site were gifts from Drs. Don Ganem and Yuying Liang (Liang et al., 2002). The constitutively active Notch expression plasmid, pcDNA-3-mNotch- $\Delta \mathrm{E}$, was a gift from Dr. Franz Oswald (Oswald et al., 2002). CMV- $\beta$-gal and CMV-GFP expression plasmids were from Clontech. Peptide
RTA antibody was described (Xu et al., 2007). Tubulin and FLAG-antibodies were purchased from Sigma. HA and RBP$\mathrm{J} \kappa$ antibodies were purchased from Santa Cruz.

\subsection{Western blot analysis, cell culture, transient transfection and reporter assays}

Standard western blot analysis was performed as previously described (Zhang and Pagano, 1997; Zhang and Pagano, 1999; Zhang and Pagano, 2000; Zhang and Pagano, 2001). 293-Bac (a gift from Dr. S.J. Gao) is a human 293 cells derived cell line and containing KSHV genome (Zhou et al., 2002). 293Bac36 cells were maintained in DMEM plus $10 \%$ fetal bovine serum (FBS) plus $0.5 \mathrm{mg} / \mathrm{ml}$ hygromycin. 293T cells are a human fibroblast line and were maintained in DMEM plus $10 \%$ FBS. Effectene (Qiagen) was used for the transfection of these cells. The luciferase assays were performed using a kit from Promega according to manufacturer's recommendation.

\subsection{Preparation of KSHV stocks and detection of virion DNA}

293-Bac36 cells were transfected with the designated expression plasmids and culture supernatants were harvested 5 days later. Virions were pelleted by centrifugation at $100,000 \times g$ for $1 \mathrm{~h}$. The pellets were subsequently suspended in $1 \times$ PBS buffer (1:100 of the volume of the original supernatants). The concentrated viruses were then treated by DNase I at $37^{\circ} \mathrm{C}$ for $1 \mathrm{~h}$. DNA was extracted and PCR analyses were carried out with primers targeted $\mathrm{K} 14 / \mathrm{vGCR}$ region (BC1 KSHV genome coordinates: 127,649-127,883). The conditions of the PCR assay were established empirically to ensure a linear amplification of template DNA within the amplification conditions.

\subsection{Chromatin immunoprecipitation (ChIP)}

293-Bac36 cells were transfected with various plasmids. One day later, ChIP assays were performed using the chromatin immunoprecipitation assay kits according to the manufacturer's recommendation (Upstate, Inc.). Anti-FLAG and normal rabbit serum (NRS) was used as designated. The DNA exacted from immunoprecipitates was used as template for PCR analyses with various primers that amplify MTA- and PANspecific products (Wang et al., 2003a; Wang et al., 2003b). PCR products were resolved in $8 \%$ polyacrylamide gel.

\subsection{Co-immunoprecipitation (Co-IP)}

$293 \mathrm{~T}$ cells grown in $10 \mathrm{~cm}$ plates were transfected with the designated plasmids. Cell extracts were prepared using RIPA buffer $(1 \times$ PBS, $1 \%$ NP- $40,0.5 \%$ sodium deoxycholate, $0.1 \%$ SDS) supplemented with a protease inhibitor cocktail (Roche) were incubated with either mouse anti-FLAG monoclonal antibody M2 (Sigma) or anti-HA antibody (Santa Cruz) for $1 \mathrm{~h}$ on ice. Protein G-sepharose (Pharmacia) was added and the incubation continued at $4{ }^{\circ} \mathrm{C}$ overnight with gentle rotation. Beads were washed three times with $1 \times$ PBS buffer and boiled in SDS loading buffer and subsequent western blot were essentially the same as described. 


\subsection{Electrophoretic mobility shift assay (EMSA)}

The probes were obtained by first annealing complementary oligonucleotides and then labeling them with $\left(\alpha-{ }^{32} \mathrm{P}\right) \mathrm{dCTP}$ (Amersham) using DNA polymerase Klenow fragment (Fermentas). The RBP-Jк probe was obtained by annealing two oligonucleotides, 5'-GATCTGGTGTAAACAC GCCGTGGGAAAAAATTTATG-3' and its complementary stand, with GATC at the 5' end. DNA-binding reactions were performed essentially as described (Liang et al., 2002; Lukac et $a l ., 2001)$ with the following modifications: the reaction buffer contained $10 \mathrm{mM}$ Tris- $\mathrm{HCl}$ (pH 7.5), $50 \mathrm{mM} \mathrm{NaCl}, 5.3 \mathrm{mM}$ $\mathrm{MgCl}_{2}, 1 \mathrm{mM}$ EDTA, $1 \mu \mathrm{g}$ polydIdC and $4 \%$ glycerol and the reactions were carried out at $25^{\circ} \mathrm{C}$ for $30 \mathrm{~min}$. If an antibody was used for a super-shift experiment, $1 \mu \mathrm{l}$ of the target antibody was added into the reaction mixture.

\subsection{In vitro transcription and translation}

Proteins were synthesized with the TNT coupled transcription and translation kit with rabbit reticulocyte lysate (Promega) according to the manufacturer's instructions. pcDNA3RTA and RTA-K152E (Zhang et al., 2005) and FLAG-RBP-Jא were used for in vitro transcription and translation of target proteins. Luc-plasmid was provided by the manufacturer as a positive control for the kit.

\section{Results}

\subsection{RTA-K152E binds to cellular RBP-JK}

The RTA-K152E mutant does not bind DNA efficiently in vitro and fails to activate several KSHV promoter reporter constructs or endogenous lytic gene expression (Zhang et al., 2005). However, it is not known whether RTA-K152E interacts with RBP-J $\kappa$. To test whether RTA-K152E interacts with RBP-J $\kappa$, plasmids expressing these proteins were transfected into 293T cells and co-immunoprecipitation assays (coIP) were performed. Expression plasmids containing wtRTA, RTA-K152E and RBP-Jא were transfected into 293T cells. The transfected RTA and RBP-J $\kappa$ were tagged by FLAG or influenza hemagglutinin (HA) epitope, respectively (see Section 2 for details). As shown in Figure 1, both wtRTA and RTAK152E interacted with RBP-JK in co-IP assays. In several experiments, wtRTA and RTA-K152E appeared to interact with RBP-JK with similar efficiency. In addition, RTA interacted with endogenous RBP-Jк in co-IP assays (data not shown). Thus, the RTA-K152E interacted with RBP-JK protein in transiently transfected $293 \mathrm{~T}$ cells.

\subsection{RTA-K152E binds to RBP-J火 site indirectly in vitro}

To test whether the complex between RTA-K152E and RBPJ $\kappa$ interacted with DNA, EMSA was performed with an RBP$\mathrm{J} \kappa$ consensus recognition sequence as a probe. The respective proteins were synthesized to similar levels by in vitro transcription and translation (Figure 2B). The expression of RBP$\mathrm{J} \kappa$ was also confirmed by western blot with the anti-FLAG

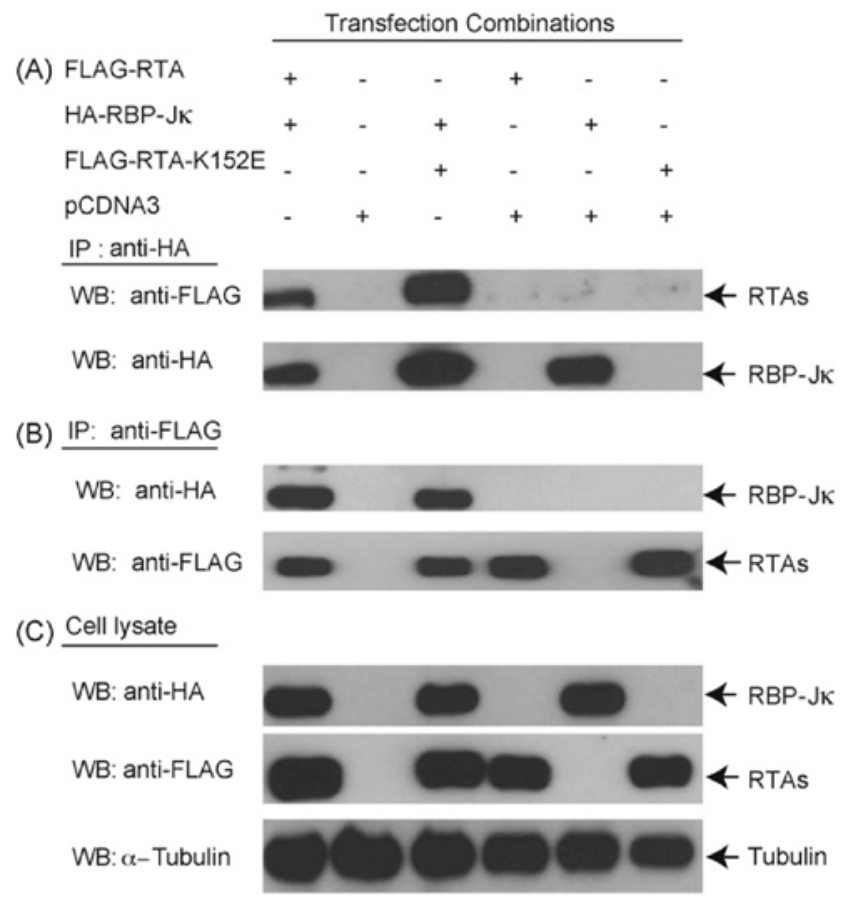

Figure 1. RTA-K152E interacts with RBP-Jк. 293T cells were transfected with the designated expression plasmids as shown on the top of the figure. Cell extracts from these transfected cells were immunoprecipitated (IP) with either anti-HA (panel A) or anti-FLAG (panel B). The immunoprecipitates were analyzed by western blot (WB) using the indicated antibodies. In panel $\mathrm{C}$, whole cell lysate was used for western blot analyses. The identity of the respective proteins is denoted.

antibody (data not shown). Various combinations of in vitro translated proteins were used for EMSA with a RBP-Jא consensus recognition sequence as probe. As shown in Figure 2, a specific shifted band representing the RBP-J $\kappa$ protein binding to the RBP-Jк probe was observed (lane 3). The shifted band was competed for by incubating with $50 \times$ of a cold wild type RBP-JK recognition sequence oligonucleotides. In contrast, a mutated RBP-JK recognition sequence oligonucleotides did not compete for binding (data not shown). The RTA or RTA-K152E proteins were unable to bind to the RBP-Jא probe directly, which was expected (lanes 4 and 5). Additional protein-DNA complexes appeared when RTA, or the RTAK152E mutant, was incubated with RBP-Jא prior to performing EMSA (lanes 6 and 7). This novel DNA-protein complexes were specific because they disappeared in the presence of a RTA antibody (lanes 8 and 9), but were not affected by normal rabbit serum (NRS) (lanes 10 and 11). Furthermore, the complexes were not present when the RTA antibody was mixed with either RBP-JK or RTA individually (lanes 12 and 13). In summary, these results suggested that both wtRTA and the RTA-K152E mutant were able to interact with the RBP$\mathrm{J} \kappa$ protein and consequently bind as a complex to a consensus RBP-JK recognition site.

\subsection{RTA-K152E binds to KSHV DNA in vivo}

To test whether a complex between RTA-K152E and RBP-Jא forms in vivo and binds DNA, ChIP assays were performed. 

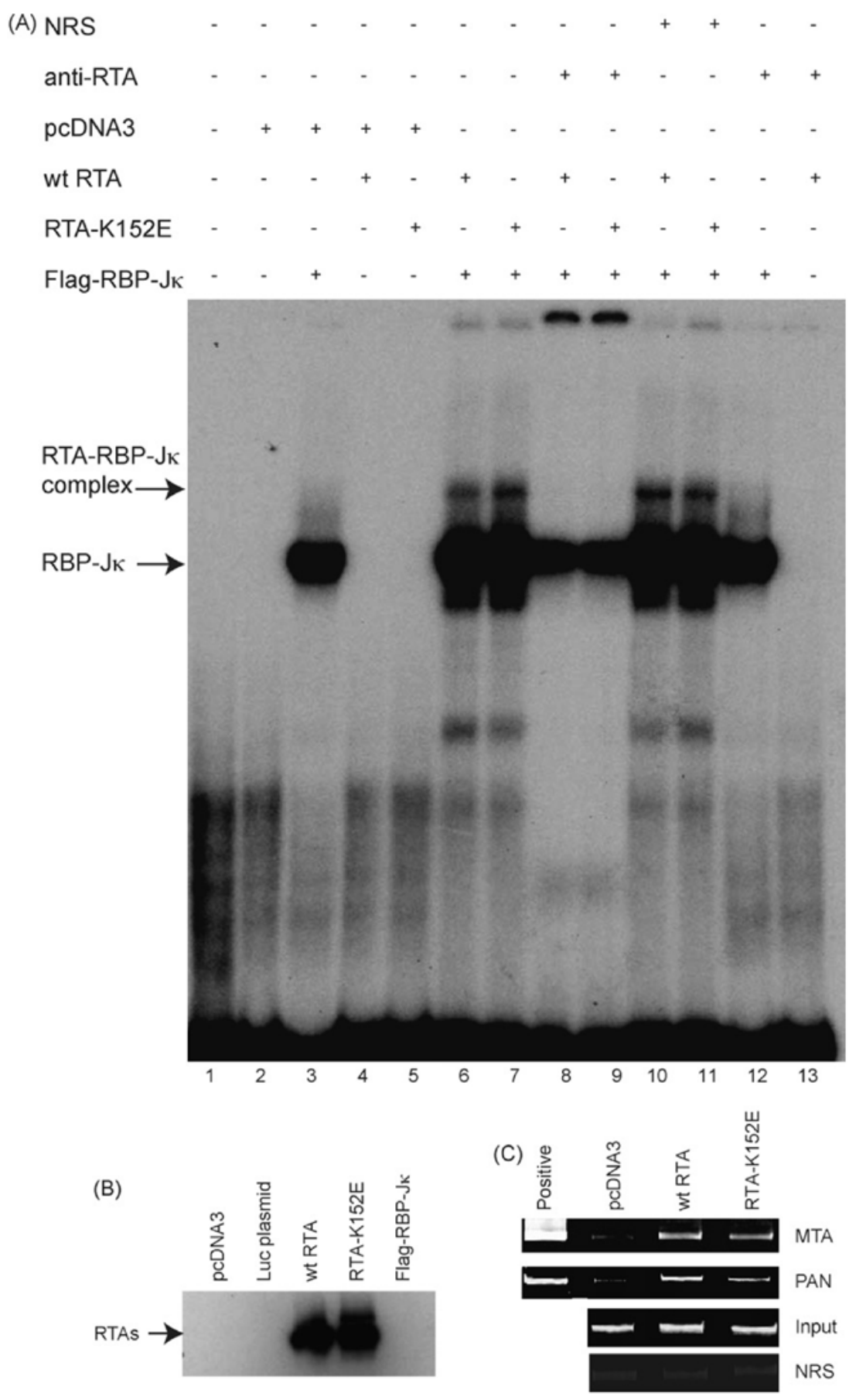

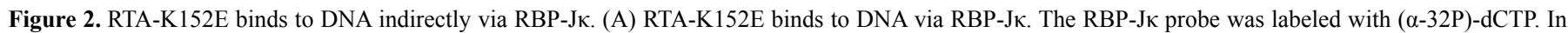
vitro transcribed and translated proteins were used for EMSA analyses in various combinations as shown on the top of the gel. Normal rabbit serum (NRS) and rabbit polyclonal anti-RTA were used for blocking the complex formations. Specific protein-DNA complexes are as shown. (B) Expression levels of RTA. Equal amounts of in vitro transcribed and translated reticulocyte lysates containing RTA and RTA-K152E proteins were used for western blot analyses. The identity of proteins is as shown. (C) RTA-K152E binds to KSHV genomic DNA in vivo. 293-Bac36 cells were transfected with pcDNA3, RTA or RTA-K152E expression plasmid. Twenty-four hours later, FLAG antibody was used for ChIP analyses to detect in vivo DNA-binding activities of RTA proteins derived from input plasmids. Primers for MTA and Pan promoter regions were used to amplify the DNA from immunoprecipitates. The identity of DNA is as shown. The genomic DNA was used as positive control. Input DNA represents the PCR amplification from same amounts of input lysates without immunoprecipitations. PCR amplification of immunoprecipitates from normal rabbit serum (NRS) is also shown as controls.

The wtRTA or RTA-K152E mutant was transfected into 293Bac36 cells, a human 293 derived cell line harboring the KSHV genome (Zhou et al., 2002). Expression of the two RTA proteins was similar (data not shown, also Figure 5B).
Anti-FLAG antibody was used for ChIP analyses because this approach avoided the endogenous auto-regulation of RTA expression (Deng et al., 2000; Sakakibara et al., 2001). Two KSHV promoter regions, MTA and PAN, were used as tar- 
gets for amplifying DNA from the immunoprecipitates as described (Wang et al., 2003a; Wang et al., 2003b). As shown in Figure 2C, both the MTA and Pan promoter regions were amplified from immunoprecipitates regardless of whether wtRTA or RTA-K152E was transfected. However, PCR amplification resulted in the similar amounts of target DNA from the immunoprecipitates by normal rabbit serum (NRS). In addition, input DNA before immunoprecipitations was similar. These results collectively suggested that both wtRTA and RTA-K152E interacted with KSHV genomic DNA in 293T cells with similar efficiencies, which was in agreement with the EMSA data (Figure 2A).

\subsection{The RTA-K152E does not activate transcription, in spite of interacting with RBP-JK}

wtRTA interacts with the RBP-Jא protein and consequently activates specific viral promoters (Carroll et al., 2006; Liang et al., 2002; Liang and Ganem, 2003). To test whether the RTA-K152E mutant was capable of trans-activating viral transcription, the ability of the RTA-K152E mutant to activate a minimal promoter construct containing multiple RBP$\mathrm{J} \kappa$ consensus binding sequences was examined (Liang et al., 2002). The promoter reporter construct and RTA expression plasmids were co-transfected into 293T cells and reporter activities measured. As shown in Figure 3A, wtRTA was able to activate the minimum promoter reporter construct containing RBP-JK-binding sites approximately 15 -fold in a dose-dependent fashion. In sharp contrast, the RTAK152E failed to activate the reporter construct above basal levels. The mutant RTA was expressed in relatively higher amounts

(A)

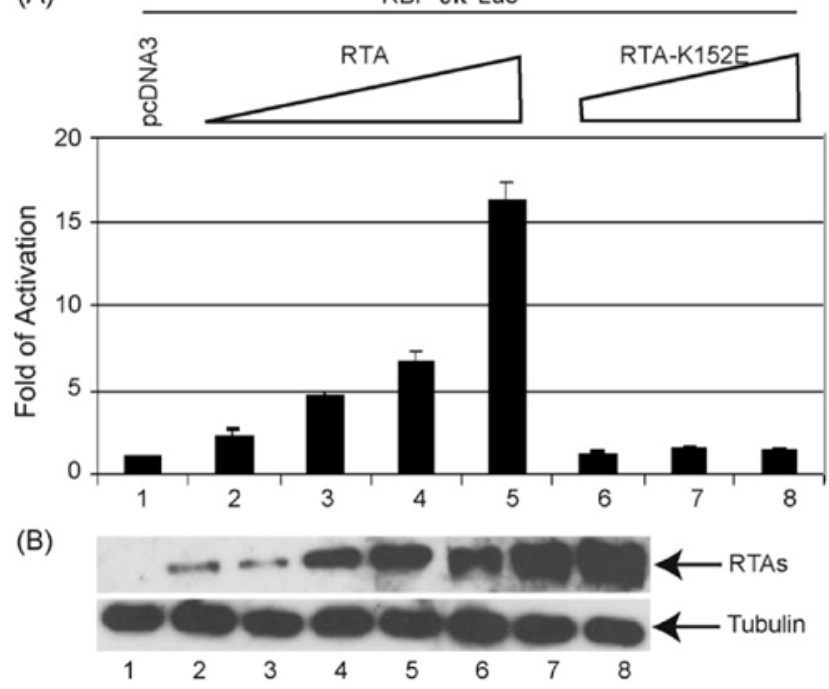

Figure 3. RTA-K152E fails to activate RBP-Jк protein. (A) RTA-K152E fails

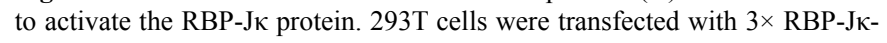
Luc reporter constructs along with CMV- $\beta$-gal, RTA or RTA-K152E expression plasmids as shown on the top of the panel. Luciferase activity was normalized by $\beta$-galactosidase activity. The fold activation of promoter constructs in response to K-RTA is shown with standard deviations. The results from one representative experiment in triplicates were shown. (B) Expression levels of RTA and its mutant. Western blotting with FLAG antibody was performed. The cell lysate from transfected cells are indicated. The identity of proteins is as shown. Lane numbers denote the same treatment as shown in panel A. than wtRTA (Figure 3B). Because comparable $\beta$-galactosidase activities were observed between wtRTA and the mutant RTA expressing cells, there was no apparent toxicity associated with mutant RTA expressed cells. In addition, same results could be obtained with the similar levels of RTAs by manipulation of transfected plasmid amounts (data not shown). All these results suggested that RTA-K152E was unable to convert RBP-J $\mathrm{\kappa}$ protein into a transcriptional activator.

\subsection{RTA-K152E interferes intracellular Notch signaling}

To test the physical interaction between the mutant RTA and RBP-JK from another direction, we examined if RTA-K152E affects the Notch signaling pathway. Because RBP-JK is involved in the Notch signaling pathway, we suspect that overexpression of the RTA-K152E mutant would alter the Notch signaling pathway by interacting and sequestering the RBP$\mathrm{J} \kappa$ protein. A constitutive active mutant of Notch was used for these studies. As shown in Figure 4, the constitutively active Notch mutant activated the RBP-JK reporter construct by at least 20 -fold in a dose-dependent fashion. The expression of wtRTA-stimulated the RBP-J $\kappa$ reporter construct more than 40-fold. The co-expression of wtRTA with activated Notch did not further stimulate the RBP-JK reporter construct activ-

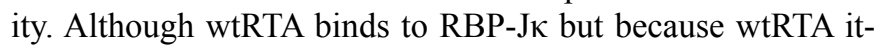
self activates RBP-J $\kappa$, no effect of wtRTA on Notch signaling pathway is anticipated. In sharp contrast, the RTA-K152E mutant interfered with the ability of Notch to activate the RBP$\mathrm{J} \kappa$ promoter construct. Due to the fact that mutant RTA interacts with RBP-J $\kappa$, these data suggest that mutant RTA inhibit the Notch signaling and possibly through sequestering RBP$\mathrm{J}_{\kappa}$. These data indirectly support the physical interaction between the mutant RTA and RBP-JK in vivo.

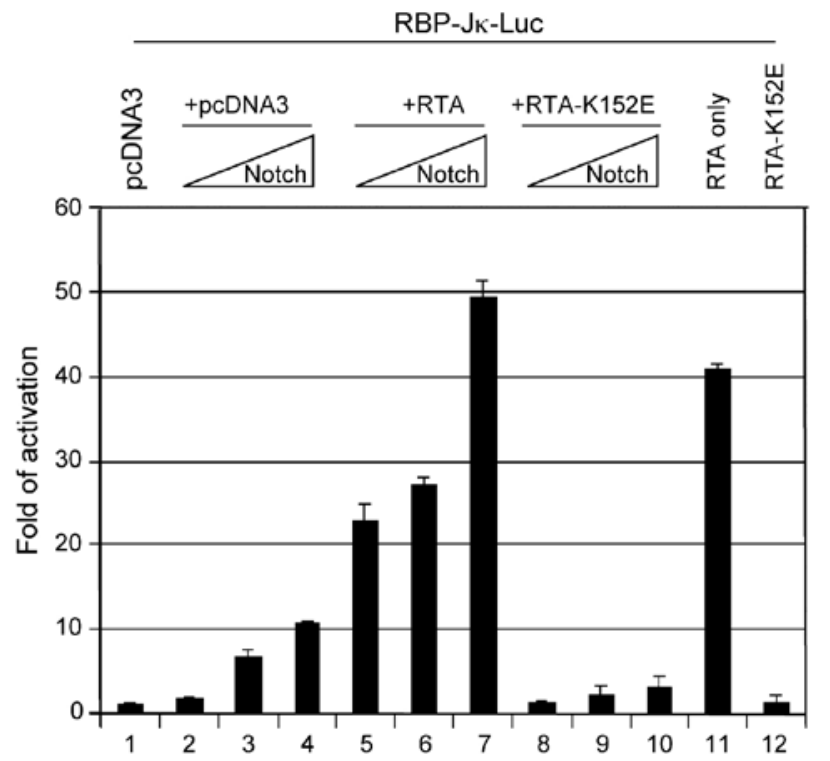

Figure 4. RTA-K152E interferes intracellular Notch signaling. Various amounts of constitutively active Notch were transfected into 293T cells with a fixed amount of the other plasmids as shown on the top of the panel. Luciferase activity was normalized by $\beta$-galactosidase activity. The fold activation of promoter constructs was shown with standard deviations. The results from one representative experiment in triplicates were shown. 

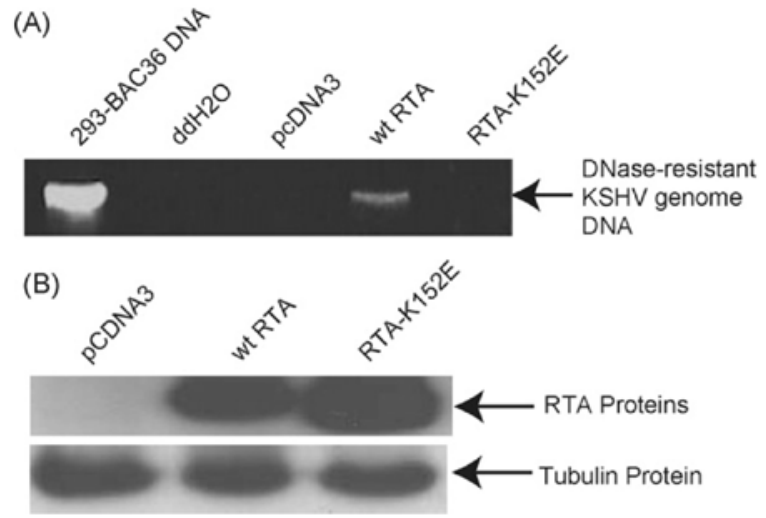

Figure 5. RTA-K152E fails to induce KSHV virion production. (A) RTAK152E fails to induce DNase-resistant KSHV genomic DNA packaged into virions. The media from pcDNA3, RTA or RTA-K152E transfected 293-Bac36 cells were collected and KSHV viruses were concentrated by centrifugation. After DNase I digestion, PCR analyses were used for detecting KSHV genomic DNA. The genomic DNA isolated from 293-Bac36 was used as positive control. (B) Expression of RTA and its mutant in transfected cells. Lysate from pcDNA3, RTA, or RTA-K152E transfected 293-Bac36 cells were used for western blot analysis with FLAG and tubulin antibodies. The identity of the respective proteins is denoted.

\subsection{RTA-K152E fails to induce KSHV virion production}

We have previously shown that RTA-K152E fails to activate KSHV lytic genes through direct DNA binding (Zhang et al., 2005). In this report, data in Figure 3 and Figure 4 suggested that RTA-K152E would not activate genes mediated by RTARBP-JK-complex through an indirect DNA-binding mechanism. Because RTA activates viral promoters through both direct and indirect DNA-binding mechanism, it is thus necessary to directly test if the mutant fails to induce a complete KSHV lytic replication. KSHV virion production is the final step of the lytic replication and we thus measured the production of the virions in response to RTA expression. The expression plasmid of wtRTA or RTA-K152E mutant was transfected into 293-Bac36 cells (Zhou et al., 2002). The expression levels of RTAs were similar in cell lysates (Figure 5B). The media were collected 5 days later and virus particles in the media were concentrated and digested by DNase. Semi-quantitative PCR was subsequently used to detect the DNase-resistant KSHV genomic DNA packaged in virions. The condition of the PCR was established empirically for a linear amplification of template DNA. As expected, wtRTA induced the accumulation of DNase-resistant KSHV genomic DNA (Figure $5 \mathrm{~A}$ ). In contrast, DNase-resistant genomic DNA was not detected following transfection of 293-Bac36 cells with the RTA-K152E mutant. Thus, RTA-K152E mutant was unable to induce KSHV virion production.

\section{Discussion}

Numerous studies have indicated that RTA activates target promoters by directly binding to DNA and by indirectly binding via interaction with various cellular factors (Dourmishev et al., 2003; West and Wood, 2003). RBP-Jк plays an essen- tial role in RTA-mediated KSHV lytic replication. In this report, we have addressed whether the RTA-RBP-Jא interaction is sufficient for the lytic replication of KSHV.

Notch signaling is well conserved in evolution. Many viruses usurp this signaling pathway for their own benefits (Hayward, 2004). However, none of them, including KSHV, can be completely replaced by cellular Notch signaling (Chang et al., 2005). RTA must have its own specific properties to usurp Notch signaling pathway. RTA-K152E has a mutated DNA-binding domain and has a defect in its direct DNA-binding activity as determined by EMSA with the purified protein. Like wtRTA, RTA-K152E interacted with RBP$\mathrm{J}_{\kappa}$ physically and the mutant-RBP-JK complex was able to bind to DNA (Figure 1 and Figure 2). However unlike the wtRTA, the physical interaction between the RTA mutant and

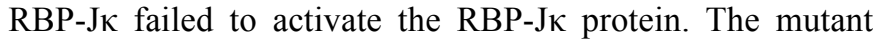
further inhibited the function of a constitutively active Notch mutant (Figure 4). Moreover, as an extension to our previous publication, the RTA-K152E failed to induce KSHV virion accumulation (Figure 5). Thus, two apparently contradicting properties of RTA-K152E have clearly emerged from our data: (1) RTA-K152E failed to activate the RBP-Jк protein; but (2) the mutant had similar capabilities to interact with RBP-J $\kappa$ and furthermore to bind to DNA indirectly via the interaction. The two properties collectively suggest that the interaction between RTA and RBP-JK and their subsequent DNA binding by the complex were not sufficient for the initiation of lytic replication of KSHV.

It remains unclear how the mutation in the DNA-binding domain of RTA had such a profound effect on RTA functions. Because of the known defect in this mutant, our results might suggest that direct DNA-binding activity of RTA might be required to convert the RBP-J $\kappa$ protein from a repressor into an activator. Since RTA interacts with many cellular factors (Carroll et al., 2006; Gwack et al., 2001; Gwack et al., 2002; Liang et al., 2002; Liang and Ganem, 2003; Liao et al., 2003; Wang et al., 2001), it is possible that the K152E mutation affects the binding of an unknown cellular factor. It is also possible that the mutation at the DNA-binding domain (K152E) has changed RTA conformation from a transcriptional activator into a repressor. Based on the structure and function analysis of RTA (Dourmishev et al., 2003; West and Wood, 2003), the activation domain is located at the $\mathrm{C}$-terminal region of the RTA. Thus, it is hard to imagine that a single point mutation at the $\mathrm{N}$-terminus would affect the $\mathrm{C}$-terminal activation domain.

In summary, this report examined the physical interaction between RTA and RBP-J $\kappa$ with respect to the activation of the RBP-JK protein and the activation of KSHV lytic replication. Our data demonstrated that the physical interaction between KSHV RTA and RBP-JK and their subsequent DNA binding by the complex were not sufficient to activate RBP-JK and to induce KSHV lytic replication. Other factor(s), whether it is the intrinsic property of RTA or its interaction with other cofactors, are predicted to be required for the activation of RBP$\mathrm{J} \kappa$ protein by RTA. These findings have provided a new insight into the structure and functions of RTA. 
Acknowledgements: We thank Drs. Shou-Jiang Gao, Don Ganem, Paul Ling, Yuying Liang and Franz Oswald for providing various reagents for this work. Supported in part by grants from the National Institute of Health AI59132, CA108951, P20RR15635 and U54 AI057160 (LZ). TC was supported by NIH Ruth L. Kirschstein fellowship (T32 AIO60547).

\section{References}

BRAY, 2006 S.J. Bray, Notch signalling: a simple pathway becomes complex, Nat. Rev. Mol. Cell Biol. 7 (9) (2006), pp. 678-689.

Carroll et al., 2006 K.D. Carroll, W. Bu, D. Palmeri, S. Spadavecchia, S.J. Lynch, S.A. Marras, S. Tyagi and D.M. Lukac, Kaposi's sarcomaassociated herpesvirus lytic switch protein stimulates DNA binding of RBP-JK/CSL to activate the Notch pathway, J. Virol. 80 (19) (2006), pp. 9697-9709.

Chang And Moore, 1996 Y. Chang and P.S. Moore, Kaposi's sarcoma (KS)associated herpesvirus and its role in KS, Infect. Agents Dis. 5 (4) (1996), pp. 215-222.

Chang ET AL., 1994 Y. Chang, E. Cesarman, M.S. Pessin, F. Lee, J. Culpepper, D.M. Knowles and P.S. Moore, Identification of herpesvirus-like DNA sequences in AIDS-associated Kaposi's sarcoma, Science 266 (5192) (1994), pp. 1865-1869.

Chang ET AL., 2005 H. Chang, D.P. Dittmer, Y.C. Shin, Y. Hong and J.U. Jung, Role of Notch signal transduction in Kaposi's sarcoma-associated herpesvirus gene expression, J. Virol. 79 (22) (2005), pp. 14371-14382.

Deng ET AL., $2000 \mathrm{H}$. Deng, A. Young and R. Sun, Auto-activation of the rta gene of human herpesvirus-8/Kaposi's sarcoma-associated herpesvirus, J. Gen. Virol. 81 (Pt 12) (2000), pp. 3043-3048.

Dourmishev et aL., 2003 L.A. Dourmishev, A.L. Dourmishev, D. Palmeri, R.A. Schwartz and D.M. Lukac, Molecular genetics of Kaposi's sarcoma-associated herpesvirus (human herpesvirus-8) epidemiology and pathogenesis, Microbiol. Mol. Biol. Rev. 67 (2) (2003), pp. 175-212 table of contents.

Gradoville et aL., 2000 L. Gradoville, J. Gerlach, E. Grogan, D. Shedd, S. Nikiforow, C. Metroka and G. Miller, Kaposi's sarcoma-associated herpesvirus open reading frame 50/Rta protein activates the entire viral lytic cycle in the HH-B2 primary effusion lymphoma cell line, J. Virol. $\mathbf{7 4}$ (13) (2000), pp. 6207-6212.

GwaCk ET AL., 2001 Y. Gwack, H. Byun, S. Hwang, C. Lim and J. Choe, CREB-binding protein and histone deacetylase regulate the transcriptional activity of Kaposi's sarcoma-associated herpesvirus open reading frame 50, J. Virol. 75 (4) (2001), pp. 1909-1917.

GwaCK ET AL., 2002 Y. Gwack, S. Hwang, C. Lim, Y.S. Won, C.H. Lee and J. Choe, Kaposi's sarcoma-associated herpesvirus open reading frame 50 stimulates the transcriptional activity of STAT3, J. Biol. Chem. 277 (8) (2002), pp. 6438-6442.

HAYwARD, 2004 S.D. Hayward, Viral interactions with the Notch pathway, Semin. Cancer Biol. 14 (5) (2004), pp. 387-396.

He and Pear, 2003 Y. He and W.S. Pear, Notch signalling in B cells, Semin. Cell Dev. Biol. 14 (2) (2003), pp. 135-142.

Liang and Ganem, 2003 Y. Liang and D. Ganem, Lytic but not latent infection by Kaposi's sarcoma-associated herpesvirus requires host CSL protein, the mediator of Notch signaling, Proc. Natl. Acad. Sci. U.S.A. 100 (14) (2003), pp. 8490-8495.

Liang ET AL., 2002 Y. Liang, J. Chang, S.J. Lynch, D.M. Lukac and D. Ganem, The lytic switch protein of KSHV activates gene expression via functional interaction with RBP-Jkappa (CSL), the target of the Notch signaling pathway, Genes Dev. 16 (15) (2002), pp. 1977-1989.

Liao ET AL., 2003 W. Liao, Y. Tang, S.F. Lin, H.J. Kung and C.Z. Giam, KbZIP of Kaposi's sarcoma-associated herpesvirus/human herpesvirus 8 (KSHV/HHV-8) binds KSHV/HHV-8 Rta and represses Rta-mediated transactivation, J. Virol. 77 (6) (2003), pp. 3809-3815.

LUKAC ET AL., 1999 D.M. Lukac, J.R. Kirshner and D. Ganem, Transcriptional activation by the product of open reading frame 50 of Kaposi's sarcomaassociated herpesvirus is required for lytic viral reactivation in B cells, $J$. Virol. 73 (11) (1999), pp. 9348-9361.

LuKaC ET AL., 2001 D.M. Lukac, L. Garibyan, J.R. Kirshner, D. Palmeri and D. Ganem, DNA binding by Kaposi's sarcoma-associated herpesvirus lytic switch protein is necessary for transcriptional activation of two viral delayed early promoters, J. Virol. 75 (15) (2001), pp. 6786-6799.

Moore and Chang, 2001 P. Moore and Y. Chang, Kaposi's sacrcoma-associated herpesvirus. In: D.M. Knipe, Editor, Virology, Lippincott Williams and Wilkins, Philadelphia (2001), pp. 2803-2833.
Oswald ET AL., 2002 F. Oswald, U. Kostezka, K. Astrahantseff, S. Bourteele, K. Dillinger, U. Zechner, L. Ludwig, M. Wilda, H. Hameister, W. Knochel, S. Liptay and R.M. Schmid, SHARP is a novel component of the Notch/RBP-Jkappa signalling pathway, EMBO J. 21 (20) (2002), pp. 5417-5426.

SAKakibara $E T A L ., 2001$ S. Sakakibara, K. Ueda, J. Chen, T. Okuno and K. Yamanishi, Octamer-binding sequence is a key element for the autoregulation of Kaposi's sarcoma-associated herpesvirus ORF50/Lyta gene expression, J. Virol. 75 (15) (2001), pp. 6894-6900.

SARID ET AL., 1998 R. Sarid, O. Flore, R.A. Bohenzky, Y. Chang and P.S. Moore, Transcription mapping of the Kaposi's sarcoma-associated herpesvirus (human herpesvirus 8) genome in a body cavity-based lymphoma cell line (BC-1), J. Virol. 72 (2) (1998), pp. 1005-1012.

Saveliev ET AL., 2002 A. Saveliev, F. Zhu and Y. Yuan, Transcription mapping and expression patterns of genes in the major immediate-early region of Kaposi's sarcoma-associated herpesvirus, Virology 299 (2) (2002), pp. 301-314.

Sun ET AL., 1998 R. Sun, S.F. Lin, L. Gradoville, Y. Yuan, F. Zhu and G. Miller, A viral gene that activates lytic cycle expression of Kaposi's sarcoma-associated herpesvirus, Proc. Natl. Acad. Sci. U.S.A. 95 (18) (1998), pp. 10866-10871.

Sun ET AL., 1999 R. Sun, S.F. Lin, K. Staskus, L. Gradoville, E. Grogan, A. Haase and G. Miller, Kinetics of Kaposi's sarcoma-associated herpesvirus gene expression, J. Virol. 73 (3) (1999), pp. 2232-2242.

WANG ET AL., 2001 S. Wang, S. Liu, M.H. Wu, Y. Geng and C. Wood, Identification of a cellular protein that interacts and synergizes with the RTA (ORF50) protein of Kaposi's sarcoma-associated herpesvirus in transcriptional activation, J. Virol. 75 (24) (2001), pp. 11961-11973.

Wang ET AL., 2003a S.E. Wang, F.Y. Wu, M. Fujimuro, J. Zong, S.D. Hayward and G.S. Hayward, Role of CCAAT/enhancer-binding protein alpha (C/ EBPalpha) in activation of the Kaposi's sarcoma-associated herpesvirus (KSHV) lytic-cycle replication-associated protein (RAP) promoter in cooperation with the KSHV replication and transcription activator (RTA) and RAP, J. Virol. 77 (1) (2003), pp. 600-623.

WANG $E T A L$., 2003b S.E. Wang, F.Y. Wu, Y. Yu and G.S. Hayward, CCAAT/ enhancer-binding protein-alpha is induced during the early stages of Kaposi's sarcoma-associated herpesvirus (KSHV) lytic cycle reactivation and together with the KSHV replication and transcription activator (RTA) cooperatively stimulates the viral RTA, MTA and PAN promoters, J. Virol. 77 (17) (2003), pp. 9590-9612.

WeST AND Wood, 2003 J.T. West and C. Wood, The role of Kaposi's sarcomaassociated herpesvirus/human herpesvirus-8 regulator of transcription activation (RTA) in control of gene expression, Oncogene 22 (33) (2003), pp. 5150-5163.

Xu $E T$ AL., 2007 D. Xu, T. Coleman, J. Zhang, A. Fagot, C. Kotalik, L. Zhao, P. Trivedi, C. Jones and L. Zhang, Epstein-Barr virus inhibits Kaposi's sarcoma-associated herpesvirus lytic replication in primary effusion lymphomas, J. Virol. 81 (2007), pp. 6068-6078.

Zhang and Pagano, 1997 L. Zhang and J.S. Pagano, IRF-7, a new interferon regulatory factor associated with Epstein-Barr virus latency, Mol. Cell Biol. 17 (1997), pp. 5748-5757.

Zhang and Pagano, 1999 L. Zhang and J.S. Pagano, Interferon regulatory factor 2 represses the Epstein-Barr virus BamH I Q latency promoter in type III latency, Mol. Cell Biol. 19 (1999), pp. 3216-3223.

Zhang and Pagano, 2000 L. Zhang and J.S. Pagano, Interferon regulatory factor 7 is induced by Epstein-Barr virus latent membrane protein 1, $J$. Virol. 74 (2000), pp. 1061-1068.

Zhang and Pagano, 2001 L. Zhang and J.S. Pagano, Interferon regulatory factor 7 mediates the activation of Tap-2 by Epstein-Barr virus latent membrane protein 1, J. Virol. 75 (2001), pp. 341-350.

Zhang ET AL., 2005 J. Zhang, J. Wang, C. Wood, D. Xu and L. Zhang, Kaposi's sarcoma-associated herpesvirus/human herpesvirus 8 replication and transcription activator regulates viral and cellular genes via interferonstimulated response elements, J. Virol. 79 (2005), pp. 5640-5652.

Zноu ET AL., 2002 F.C. Zhou, Y.J. Zhang, J.H. Deng, X.P. Wang, H.Y. Pan, E. Hettler and S.J. Gao, Efficient infection by a recombinant Kaposi's sarcoma-associated herpesvirus cloned in a bacterial artificial chromosome: application for genetic analysis, J. Virol. 76 (12) (2002), pp. 6185-6196.

ZHU ET AL., 1999 F.X. Zhu, T. Cusano and Y. Yuan, Identification of the immediate-early transcripts of Kaposi's sarcoma-associated herpesvirus, $J$. Virol. 73 (7) (1999), pp. 5556-5567. 